Voix et Images

voixetimages

\title{
" Le Tombeau des rois » ou la progression régressive
}

\section{Pierre Kunstmann}

Volume 2, numéro 2, décembre 1976

Paul Chamberland

URI : https://id.erudit.org/iderudit/200057ar

DOI : https://doi.org/10.7202/200057ar

Aller au sommaire du numéro

Éditeur(s)

Les Presses de l'Université du Québec

\section{ISSN}

0318-9201 (imprimé)

1705-933X (numérique)

Découvrir la revue

Citer cet article

Kunstmann, P. (1976). « Le Tombeau des rois » ou la progression régressive.

Voix et Images, 2(2), 255-264. https://doi.org/10.7202/200057ar d'utilisation que vous pouvez consulter en ligne.

https://apropos.erudit.org/fr/usagers/politique-dutilisation/ 


\section{«Le Tombeau des rois» ou la progression régressive}

Les réflexions que je présente ${ }^{1}$ sont nées à l'occasion d'une explication de texte avec mes étudiants de premier cycle et à la lecture d'un pénétrant commentaire de $P$. Lemieux ${ }^{2}$ qui, ne prétendant point épuiser la richesse de ce poème-titre, offre cependant une base solide pour toute étude complémentaire. L'analyse qui suit ne veut nullement expliquer la personnalité de l'auteur; l'entreprise, inconvenante, serait vouée à l'échec: on n'ignore pas, Valéry y a suffisamment insisté, que l'œuvre, loin de toujours refléter fidèlement le soi de l'écrivain, peut au contraire remplir une fonction compensatoire: "la Jeune Parque» constitua ainsi un exercice de discipline poétique pour un esprit plongé dans l'angoisse quotidienne du temps de guerre.

II importe donc, sur le seuil, de décevoir d'emblée le lecteur: il ne lira pas ici une esquisse d'analyse de la psychè d'A. Hébert, mais un commentaire, dans une perspective jungienne, du réseau symbolique ${ }^{3}$ du poème qui, de l'avis de tous, forme le centre de son cuvre. Point cardinal chez notre poétesse, puisqu'il correspond à une opération essentielle de l'âme, à cette épreuve que tcut être humain doit affronter s'il veut se posséder pleinement. Les anciens parlaient de descente aux enfers, je dirai avec Jung qu'il s'agit d'une phase d'introspection de la libido. L'énergie vitale renonce temporairement au monde extérieur comme objet, pour rentrer en soi explorer un espace intérieur. Cette conversion, "régression de la libido" selon l'analyste zurichois, ramène à la prime enfance, à l'CEdipe, au sein maternel. Le flot du désir remonte à sa source pour en rejaillir moins purifié peut-être qu'enirichi. Le but visé: «devenir ce que nous sommes " suivant l'heureuse expression de Ch. Baudoin, c'est-à-dire «échapper au moi que nous nous étions forgé..., être repris par plus grand que nous et par le peuple même des ancêtres ${ }^{4}$. Ce retour à l'origine, situé par Jung entre trente-cinq et quarante ans, doit revêtir un caractère volontaire et presque méthodique si l'on ne veut pas sombrer durant la traversée. Qui réussit à sortir de ce degré zéro (point mort) auquel la régression dynamique l'a ramené, s'en trouve régénéré; les mythologies parlent à juste titre de seconde naissance - mystère du passage à l'âge d'homme, de la pause méditative au mouvement prédicatif: mystère de la parole. 
On distinguera par commodité quatre étapes dans ce processus qui, nous le verrons, participe des anciens rites d'initiation: sevrage de la réalité extérieure, catabase, affrontement, anabase. La première, elliptique dans notre poème, est cependant bien marquée, parfois même développée, en d'autres points du recueil. Le monde, dont il faut maintenant effacer la beauté ( $p .51$ ), se présente, par réduction synecdochique, comme le jour que les mains écartent tel un rideau (p. 58), comme l'éclat de midi (non le juste, mais l'ardent qui dessèche et consume, p. 45), cet excès de lumière qui empêche de voir (p. 57); c'ẹst aussi la rue conduisant à autrui, les chemins menant ailleurs auxquels renonce le moi pour se barricader dans «la plus étanche maison/ La plus creuse la plus profonde" (p. 45) ${ }^{5}$. Un mur (p. 37) se dresse, tout symbolique - «signe de mur», séparant de l'univers le moi, cerné mais forclos; cette exclusion n'apparaît pas volontaire, ne relève pas tant du moi conscient que de contraintes, provenant de la face inconsciente de la personnalité, qui lient le sujet à la mort (c'est-à-dire à la vie comme nous le verrons plus tard); le je, contrairement à l'expression de l'auteur, n'a pas noué ces liens durs, mais en est noué; l'agent du procès, «ma fidélité", se présente dans TR comme fil d'Ariane, cordon (chaîne) ombilical rattachant à l'ancêtre. Ce lieu clos, limité de fleurons, hortus conclusus, jardin secret de la vierge à la rose des enluminures médiévales, annonce le centre du labyrinthe, la chambre mortuaire des pharaons.

L'arbre de vie planté au centre de l'enclos (p. 38) nous permettra d'amorcer la deuxième phase. Quelle meilleure expression de la régres. sion contrôlée (mais ici bientôt panique) dont parle Jung que ces vers:

Retourne sur tes pas ô ma vie

Tu vois bien que la rue est fermée! (p. 45)

Le moi rentre chez lui, s'efforce de vivre à l'intérieur (p. 47) et, tout souffle retenu, visite son cceur souterrain dans le plus grand recueillement. L'exploration est menée dans le cœur et par le cœur, englobant et englobé, lieu et agent. Organe d'une grande richesse symbolique chez l'auteur par la variété des formes qui le représentent, par la valeur aussi de ce qu'il traduit. Le sujet, se crevant la poitrine, l'arrache à sa cage de bouleau blanc; l'opération s'effectuera à cœur ouvert ou plutôt à découvert (p. 65). L' "hôte coutumier/ Entre ses côtes déraciné" (p. 40) ne se laisse point dévorer sur la table sacrificielle, mais s'offre comme guide pour la traversée de l'océan nocturne; le bruit systolique correspond au rythme du phare dont la lumière surgit régulièrement pour trancher l'obscurité - «code secret» (p. 24), le chiffre se révélera à la fin de l'épreuve. Le cœur est feu, lampe dont la panse recèle vin et sang: l'énergie vitale, l'eau-de-vie qui stimule et irrigue corps et âme - analogie du don du Christ à ses disciples, laissant dans le vin, philtre d'immortalité, son sang rédempteur ${ }^{6}$. Le feu reste intérieur, la lampe n'éclaire pas au-dehors ou si peu. Ce feu n'est point celui du soleil (archétype de la conscience), de l'univers objectal, du monde des autres, mais celui de la nuit (p. 35), du 
souterrain. Les yeux du cœur qu'allume le sujet ne sont que «fleurs tremblantes"(p. 36), vite fanées par l'éclat du jour; des yeux aveuglés, les seuls dont il dispose, que le moi prend en mains (p. 36) avant de les bran-1 dir au poing; regard terne et glauque, de pierre et d'eau (p. 36 et 51), ces deux représentations archétypales de la Grande Mère - nous irons enfin au bois éveiller les grandes fontaines (p. 17)! Et roule l'héroine dans le ravin, le cceur à son cou, «Agrafé comme un oiseau fou " (p. 55)! Quel est cet oiseau imaginaire battu par l'air du temps? L'esprit, le moi qui, rejeté par la réalité à laquelle il n'a pu s'adapter, désire retourner au paradis de l'irresponsabilité enfantine:

Sur les deux rives fume mon enfance

Sable et marais mémoire fade

Que hante le cri rauque

D'oiseaux imaginaires châtiés par le vent. (p. 56)

Voici que notre oiseau nostalgique part en quête de l'entrée du gouffre:

La voix de l'oiseau

Hors de son cœur et de ses ailes rangées ailleurs

Cherche éperduement la porte de la mémoire. (p. 53)

Efforts tâtonnants en raison de sa cécité. II semble dans TR que l'entrée se découvre plutôt qu'elle n'est découverte; l'auteur du songe y aspire le téméraire. Dans "l'Envers du monde" dont je viens de citer quelques vers, l'oiseau se réduit à une voix, pur appel à la vie, qui s'affaiblira jusqu'au "taciturne" du poème que nous étudions. Pourquoi donc cet oiseau, rappel obsédant de la «Cage d'os» du cousin poète (Saint-Denys Garneau) ? Parce qu'il a toujours été dans l'inconscient collectif une image de l'âme ${ }^{7}$ :

Cette espèce de roi

Minuscule et naïf. (p. 191)

L'oiseau de proie en particulier, qui symbolise noblesse et puissance: l'aigle (pour ne souffler mot du phénix) est considéré comme un oiseau de feu, totem du feu dans certaines tribus indiennes ${ }^{a}$. Le choix du faucon ne semble pas gratuit comme il apparaîtra par la suite. Doit-on voir dans la cécité une mutilation? Peut-être, mais il s'agirait alors d'une auto-mutilation; cette privation du regard semble plutôt un exercice pour discipliner la fonction visuelle: la réduction, la soumission de l'œil permet l'introversion; il faut fermer les "yeux avides" (p. 51), les écarter de cette “lueur de braise" qui «chante à tue-tête» (p. 57) - expression qu'on serait tenté de prendre à la lettre. Que la conversion s'opère à plein pour que la personne ne reste pas

Toute liée

Sur le chemin

Le cœur dans son coffret ancien. (p. 51)

Le monde maintenant effacé, le recueillement trouvé, la «paroi du silence» (p. 44) traversée, la descente peut s'engager. Nekuia des épopées classiques, mais sans la main rassurante d'une sybille ou les éclaircissements d'un sage Virgile. Irruption dans I'hypogée royal. Bévue grossière 
que celle d'un lecteur qui se récrierait devant une momerie égyptienne à lui injustifiée! Si nous quittons les bords du Saint-Laurent pour les rives du Nil, c'est que nous atteignons un symbole investi d'une forte énergie archétypale. Quelle que soit l'origine du décor égyptien dans l'imagination de l'auteur, sa signification psychologique apparaît manifeste: le pharaon exprime l'archétype masculin du père; à l'image paternelle s'associe souvent celle du mari (le partenaire sexuel de la hiérogamie qui suit) et même du frère - c'est tout un pour les membres de ces dynasties... Le père divinisé, deus sed absconditus, phallus perdu (ou jamais obtenu), représente le trésor secret, le but sacré de la pérégrination de l'âme. Inversion curieuse par rapport à la plupart des religions, la mythologie égyptienne voit le ciel femelle et considère la terre mâle. Lieu d'enterrements, séjour des défunts:

Le monde est en ordre

Les morts dessous

Les vivants dessus. (p. 36)

Le faucon désigne-t-il Horus? Ce dieu, dont le nom signifie le Haut, l'Élevé, fut d'abord la voûte céleste, puis le faucon qui la sillonne; la statuaire le montre souvent, comme au temple d'Edfou, en faucon coiffé du pschent, symbole de la monarchie pharaonique. Que la poétesse ait vu ou non en lui le fils d'Osiris et d'Isis, l'oiseau renaissant aux derniers vers, tout comme Horpekroud (Horus l'enfant), correspond, dans l'inconscient collectif, au puer aeternus, au médiateur-sauveur qui effectue la synthèse des aspects conscients et inconscients de la personnalité.

Le temple funéraire est labyrinthe. Le passage de l'Égypte au monde grec n'étonnera point si l'on se rappelle la description par Hérodote du temple d'Amenemhat III, construit au bord du lac Moeris, modèle de tous les labyrinthes connus des anciens. Dans l'imaginaire cette figure marque un champ où s'affrontent des tensions antagonistes. Point doublement dangereux, en raison des forces destructrices qu'on risque d'y déchaîner, à cause aussi de la fascination qu'il peut exercer sur l'âme novice. Lieu clos d'une enceinte continue, dotée d'une seule entrée - caractéristique pour Varron de tout temple antique. Selon M. Brion ${ }^{9}$, le labyrinthe est à la fois entrelacs et spirale: réseau inextricable de galeries en méandres aux multiples bifurcations et impasses fatales (l'anglais maze) et, d'autre part, voie sinueuse, mais unique (labyrinth) qui mène infailliblement au centre. La traversée comporte deux phases et suppose deux buts: on vise à pénétrer au cœur, puis à en sortir. Le labyrinthe se transforme vite en prison: le premier processus trouve généralement son lieu dans le labyrinth et le second dans le maze.

On sait que le «myste» (initié) ne peut revoir la lumière du jour sans fil d'Ariane; la mémoire devient alors la faculté souveraine. Dans certaine tribu du Pacifique, le rite initiatique veut que les jeunes gens soient conduits dans une grotte sacrée; un prêtre y dessine sur le sol un lacis compliqué, puis l'efface rapidement. Le novice qui ne peut le reconstituer de mémoire est considéré comme perdu. Le monstre engloutit la proie im- 
puissante et en dévore jusqu'à "L'écho des pas". De façon très intéressante et fort significative pour l'interprétation de l'œuvre, A. Hébert renverse l'ordre attendu: le fil n'est pas donné par une amante à son bienaimé, assassin mais libérateur, pour le ramener au monde; c'est un lien dur (p. 37) qu'un ancêtre, lui aussi amoureux, a noué à la cheville de cette esclave qu'est la petite fille apeurée, hésitant au bord du gouffre, dans un espace «de plus en plus étroit» (p. 44). Le fil est cordon ombilical qui unit le nourrisson («à peine née») au nourricier - liaison vitale, non sans aspect érotique. C'est surtout le fil de la réminiscence; le passé n'est pas évoqué en anamnèse, il s'impose («Les morts me visitent» $p$. 36) et tire de tout son poids pour faire basculer la fille et la faire pénétrer par «la porte de mémoire» (p. 53) dans la nuit de la mort. Ch. Baudoin a qualifié de fil d'Ariane le processus d'individuation jungien, c'est-à-dire la formation de la personnalité par l'intégration de ses deux faces opposées; l'analyste genevois ajoute: “mais c'est bien à travers un labyrinthe qu'il est appelé à nous guider ${ }^{10}$ ".

La plongée dans l'inconscient s'effectue en songe; le rêve, en deçà de la censure refoulante, laisse vaquer le regard sur al'envers du monde":

Nous avons mangé les plus tendres feuilles du sommeil

Les songes nous ont couchées

Au sommet de l'arbre de nuit. (p. 52)

Songe, mensonge, à en croire l'adage; il s'agit bien d'une fiction dont on mentionne l'agent - «L'auteur du songe», expression qu'A. Hébert explique ainsi dans son Dialogue sur la traduction: "on voit un être fort qui fabrique et machine tout ce rêve maléfique ${ }^{11} "$. Construction imaginaire certes, mais fantasme n'est point illusion: cette mise en scène s'offre au sujet pour le guider dans les ténèbres de son âme.

Le rêveur s'est dépouillé du masque, persona qui le fige sous le regard d'autrui ; la crise réduit à l'essentiel :

L'angoisse me fait de l'ombre

Je suis nue et toute noire sous un arbre amer. (p. 43)

L'adulte claquemuré dans la chambre noire de son recueillement (p. 76) retrouve alors le petit enfant - nostalgie irrépressible. La «chambre fermée" est "Coffre clair où s'enroule mon enfance» (p. 42). Pèlerinage au vert paradis d'une époque révolue, à jamais perdue, dont on essayera vainement de raviver, de relever la "mémoire fade" (p. 56).

Je vais coudre ma robe avec ce fil perdu.

$J$ 'ai des souliers bleus

Et des yeux d'enfant

Qui ne sont pas à moi. (p. 43)

Inanité et péril de la régression à l'enfance où sombrerait le sujet, n'était ce fil conducteur qui l'amènera à rassembler ses forces, à rompre le silence et à s'épanouir en harmonie avec soi-même et donc avec autrui. 
Le rêve d'angoisse précipite l'enfant au fond de la caverne en plein enfer (quasi homonymie en langue allemande de Höhle et Hölle). Le puits aboutit à l'étage des chambres funéraires. II s'en dégage, goutte à goutte, une a odeur capiteuse" ( $p .48$ ); la confusion métaphorique de l'olfactif et du liquide aux vers 21-22 de notre poème est annoncée et comme préparée par une strophe de «la Chambre de bois" où les éléments se présentent en juxtaposition:

Je dors sur des feuilles apprivoisées

L'odeur des pins est une vieille servante aveugle

Le chant de l'eau frappe à ma tempe

Petite veine bleue rompue

Toute la rivière passe la mémoire.

Le syntagme “orages gonflés ${ }^{12}$ " (v. 21) se réécrit en menace (ou promesse) d'orages: la tourmente emporte et brise, mais d'elle jaillit aussi l'éclair qui illumine ${ }^{13}$. "Levez-vous vite, orages désirés !..." La foudre, arme de Jupiter, dieu et père par excellence, flèche phallique, symbole ambivalent d'amour et de haine, précise le caractère, marque le genre de ces espaces limités que sont dans TR les "chambres secrètes et rondes", “les lits clos», les «étuis solennels et parés». Contrairement au schéma initiatique masculin, de loin le plus répandu et le mieux connu, où le héros, engouffré dans les entrailles de la Terre-Mère, parvient à la matrice que constitue le centre et, après une exploration incestueuse comme dans la navigation nocturne d'Osiris, renaît à la lumière, c'est à la recherche de l'homme (père, mari, fils) que s'engage le sujet dans sa pérégrination psychique. La terre (hasard heureux ou correspondance voulue) est considérée masculine dans la religion égyptienne et le ciel féminin. Si l'on jouait à prêter une figure animale au monstre que l'héroïne va maintenant affronter, s'imposerait l'image du taureau, expression des forces chtoniennes, du principe actif, grand fécondateur, et point celle du dragon qui - Jung l'a admirablement montré - symbolise la mater saeva cupidinum, terrible et castratrice.

La bête vit en nous, nourrie des reliefs de notre conscience: "L'homme, souligne Santarcangeli14, est appelé à une confrontation avec lui-même au cœur-même du labyrinthe, et à un duel avec lui-même." Le monstre apparaît à l'héroïne comme son ombre, son double, personnage étrange qui la fascine et pétrifie, et dont l'apparition soudaine et violente correspond à ce que Freud appelle le retour du refoulé. Ce qui surgit sous forme hallucinatoire dans le songe se manifeste progressivement durant la thérapie: «il faut s'attendre, note Jung, à ce que la prise de conscience de l'ombre se heurte tout d'abord en cours d'analyse à la plus grande résistance de la part de l'analysé, qui ne peut souvent pas supporter l'idée que tout ce côté sombre fait partie de lui-même ${ }^{15}$ ». Cet «étranger vêtu de noir,/ Qui me ressemblait comme un frère " personnifie l'animus, l'envers de la personnalité féminine, les traits masculins qu'elle portait en soi dans son état de bisexualité primitif, qu'elle a bannis ou plutôt enfouis en son tréfonds vers la fin de l'enfance pour accorder l'exclusivité à la féminité, traits qui remontent à la surface sous la forme de symptô- 
mes morbides et qui réclament maintenant leur place au soleil de la conscience $^{16}$. L'iter perfectionis, le processus d'individuation, vise à la conjonction de l'animus et de l'anima, au conjugium solis et lunae de l'alchimie médiévale.

Ces noces prennent un caractère de hiérogamie, de viol sacré, comme dans le rite éleusinien. L'union sexuelle avec le mort récompense les longues heures que l'héroïne passe devant le miroir, seule occupation possible dans la "Vie de château» (p. 54). Le narcissisme se révèle «enchantement pervers" ( $p .54)$ quand il conduit à la perte de l'âme, mais il peut aussi mener à la restauration du perdu, à la consolidation de la personnalité; l'économie narcissique s'avère essentielle à l'émergence de l'identité primaire, puis au développement du moi ${ }^{17}$ - pour parler en termes jungiens: à la constitution du soi et à son équilibre. Ch. Baudouin a fait remarquer que certains des traits qui composent l'image de l'ombre appartiennent en propre au registre sadique-anal: ainsi son aspect agressif (I'horrible de la strophe XIII) et sa couleur noire. D'où la qualification (à certains paradoxale) de ces squelettes fantomatiques, alors que la poitrine crevée de l'héroïne garde en surface un éclat ivoirin (p. 55); à la «Cage de bouleau blanc" du corps encore vivant correspondent en creux les étuis des pharaons d'ébène - hypallage hardi, mais combien justifié psychologiquement! A rapprocher de la "poutre d'ébène» ( $p$. 31) que cherche le sujet au faîte du château fantasmatique de son enfance pour $y$ suspendre la corde, fil d'Ariane à fonction inverse, qui fera crouler la «vieille image" et le précipitera au royaume des morts. Ces sarcophages rappellent «Le lit de bois noir» (p. 40) contenant le corps de la dormeuse dans "la Chambre fermée»; l'attente du sacrifice dans ce poème préfigure la descente dans le tombeau des rois: les points communs ressortent (caractère funéraire de la pièce, séparation du cœur pris dans le feu, yeux clos, songe...); mais les différences, qui constituent plutổt sur le plan du signifiant des variantes d'un signifié commun, n'en présentent pas moins beaucoup d'intérêt: le corps féminin, vide et glacé, symbolise la terre, figure la croix dont les extrémités font naître les points cardinaux (quaternité chère à Jung); le rite ne revêt point, en apparence, la forme d'une hiérogamie, mais d'un festin sacré où l'«ami tranquille" doit, semble-t-il, dévorer le cœur et peut-être la chair de la victime. On reconnaît sans peine l'animus qui, complice, guide l'anima, cette fois-ci sans tirer de fil, mais en soufflant sur ses pas. L'imagerie sexuelle, remplacée par celle de la nutrition (la fonction sexuelle chez l'enfant naît en s'étayant sur la fonction nutritive), se dessine en filigrane dans la dernière strophe: le feu embrasera cœur et chair pour les mûrir et les "Tristes époux tranchés et perdus" se retrouveront en une conjonction nouvelle. "L'ombre de l'amour» du TR se profile déjà en cet «homme de sel à la «lente froide respiration immobile" d'un poème précédent (p. 44) - "L'amour changé en sel» (p. 56), pétrification sentimentale; l'animus, médusé, refoulé, mais point disparu, revient dans le poème final frapper à la porte de la conscience, au cœur, et, quoique moribond, couché même dans un cercueil quii le fige, cet archétype recèle assez d'énergie (de «numinosité») 
pour attirer immanquablement l'anima déroutée et satisfaire sa pulsion. Commentant le vers 25

L'immobile désir des gisants me tire,

l'auteur précise qu'il s'agit d'un «désir physique comme si on tirait un enfant par la main». L'ombre, qui n'est pas toute obscurité puisque l'héroïne voit sur la noirceur “Luire les pierres bleues incrustées" (v. 28) image de fécondité virtuelle reprise par les «plates pierres bleues sous la mer» (p. 76) de la "Naissance du pain» dans le recueil suivant - , ce double, alter ego qui signifie l'inconscient personnel, baigne dans l'inconscient collectif qui l'informe et dont les constantes en soutiennent le tissu symbolique.

Jung a constaté dans ses travaux qu'à la différence de l'anima, représentée généralement par une personne unique, l'animus est exprimé par un groupe de personnages imposants à la façon d'un aréopage. Nos pharaons (comme d'ailleurs les "filles de feux roux" de l'"Alchimie du jour ", p. 80) sont sept: chiffre magique dont on connaît l'importance dans le mythe - le tribut d'Athènes consistait en sept jeunes hommes et sept jeunes filles offerts au Minotaure (les sept femmes de Barbe-Bleue...) et dans les diverses religions: la progression vers le centre s'opère en sept degrés - sept marches pour l'escalier de cérémonie des mystères de Mithra, sept étages aux ziqqurats babyloniennes; on retrouve les mêmes étapes dans la montée de Dante au paradis. Ce chiffre évoque encore, pour un chrétien, les vertus et les vices cardinaux, (comme le rappelle A. Hébert dans «Pentecôte") les têtes du dragon de l'Apocalypse, ainsi que la très ancienne et fort curieuse légende des sept dormants que Jung mentionne en note dans ses MAS: sept fidèles d'Ephèse, pour échapper à la persécution de l'empereur Décius au $1 \|^{-}{ }^{-}$siècle, se réfugièrent dans une caverne que l'empereur fit murer; protégés de Dieu, qui désirait contrer I'hérésie de l'évêque Théodore et montrer par un miracle le bien-fondé de la croyance en la résurrection, ils y dormirent pendant deux siècles; s'étant réveillés sous le règne de Théodose III, ils proclamèrent le miracle avant de mourir quelques instants plus tard en puissante odeur de sainteté. Mahomet, frappé de cette anecdote, décida de l'intégrer au Coran pour prouver l'immortalité de l'âme.

Les morts, enfouis dans l'hypogée, ne ressuscitent pas complètement, mais à la manière du retour partiel d'Osiris à la vie, ils paraissent retrouver un souffle qui jaillit et se propage "comme on dit que le feu prend", précise l'auteur ${ }^{18}$, ajoutant que "les pharaons sont remués par le vent qui passe. II ne s'agit pas de dérangement intérieur, moral». Passivité de ces rois qui donnent l'impression de s'animer au pneuma ${ }^{19}$, à l'esprit que semble leur insuffler le faucon:

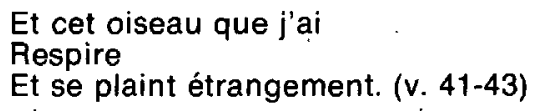

Retour en force du refoulé, l'ombre aspire à remonter à "la source fraternelle» qu'est le cœur, son double conscient; le mal qui en sourd re- 
présente les images, sentiments, désirs qu'on juge honteux et qu'on préfère enterrer au plus profond de soi, y occultant ainsi certaịns éléments de grande valeur que seule une confrontation avec l'ombre permettra de libérer. Ch. Baudoin fait remarquer la «participation homéopathique du héros à la nature du dragon ${ }^{20}$ " - ou inversement, ajouterai-je, de l'héroïne à celle du taureau. L'union prend la forme du vampirisme, véritable transfusion qui réalise la prophétie d'un poème précédent: “Mon cœur sera bu comme un fruit " (p. 38), et du cannibalisme - rompre le ccur pour le dévorer. "Celui qui mange mon corps et boit mon sang aura la vie éternelle." Communion dans le Saint (sein) des Saints; communication aussi du trésor précieux:

\section{Je reçois ton tremblement}

Comme un don 21 (p. 34)

s'écriait la "fille maigre» dans le poème de ce nom, blottie dans la poitrine de son amant, «reliquaire d'argent " où elle s'était pendue «A la place de son cœur absent»; morte en quête de renaissance dans le corps de l'autre bien vivant, elie entrouvre parfois les yeux sur «Des songes bizarres et enfantins" (p. 34). Inversement dans TR, c'est à une héroïne encore vivante que les momies attachent leur fil, espoir de retour à la vie consciente. Mais les revenants, ne pouvant sortir de leur mort, en sont réduits à déguiser et feindre: simulacre d'amour, impuissance, "tumulte des grands morts friables et irrités (p. 83).

Le moi, «livide» mais «repu», régénéré au contact des forces telluriques dont il a su mater la révolte comme jadis les adeptes du culte de Mithra sacrifiant le taureau dans la caverne («Et les morts hors de moi assassinés" v. 61), le moi qui vient d'assurer sa domination sur l'ombre peut enfin commencer son anabase. Tel un dieu pelé et victorieux (p. 91), le soleil se lève, ayant défait la nuit qui l'a restauré. "Le jour, pour la seconde fois convoqué, monte en parole comme un large pavot éclatant sur sa tige", chantera, plus tard A. Hébert (p. 83). Le plomb est transmué en or. Le retour à travers le maze de la vie s'effectuera lentement et difficilement: le souterrain ne s'ouvre que sur un "reflet d'ombre» égaré; mais la régression, stérile encore dans les premiers poèmes (“Inventaire», "Vieille Image", "la Fille maigre", "la Chambre fermée "...), s'avère cette fois-ci féconde: l'oiseau de l'âme «tourne vers le matin/ Ses prunelles crevées" ( $v$. 64-65). Les paupières cousues du faucon se dessillent, l'énergie vitale se libère des fixations antérieures, la fonction refoulée se trouve récupérée, les membres se dénouent ( "on a enlevé des liens qui attachaient les membres", commente A. Hébert, ces liens durs noués avec la mort) moment salutaire de la naissance de l'enfant de l'analyse: le puer aeternus, filius sapientiae, le jeune Horus, fruit de l'union d'Isis et d'Osiris, l'enfant de Bethléem ( «maison du pain ${ }^{22}$ ), l'apparition de l'épi d'Eleusis, la "naissance du pain». Le soi enfin équilibré, la personnalité mûrie peut s'exposer au "présent redoutable» (p. 28). Mysterium tremendum atque jocosum. Haut mystère de la parole - l'animus de la femme exprime son logos. 
Ah nous sommes vivants, et le jour recommence à l'horizon! Dieu peut naître à son tour, enfant blême, au bord des saisons mis en croix; notre œuvre est déjà levée, colorée et poignante d'odeur!» (p. 79)

Pierre Kunstmann Université d'Ottawa

1. Les références qui suivent les citations d'A. Hébert correspondent à la pagination des Poemes, Paris, Éditions du Seuil, 1960. J'utilise les abréviations TR pour "le Tombeau des rois", MP pour "Mystère de la parole"; et MAS pour Métamorphoses de l'áme et ses symboles de C.G. Jung, Georg, 1973.

2. Revue de I'Universite d'Ottawa, vol. $45, n^{\circ} 2$, p. 133-161.

3. Je rappelle avec Ch. Baudoin la présence fondamentale de l'archétype sousjacent au symbole: « $A$ la base de tout symbole se trouve un archétype qui en est la préfiguration possible; tout symbole est Pexpression d'un archétype dans une situation concrète, collective ou individuelle. " ( $L$ 'OEuvre de Jung et la psychologie complexe, Paris, Payot, 1963, p. 185)

4. Ibid., p. 151.

5. A. Thério a fait remarquer la grande importance du thème de la clôture, en particulier de la maison (cabane, château...) dans les romans d'A. Hébert ( $L i$ vres et auteurs québécois, 1971, p. 274). Voir aussi les articles de J. Féral, D. Bouchard et G.-P. Ouellette dans Voix et images, vol. 1, nos 2-3.

6. MAS, p. 289.

7. Ibid., p. 360.

8. Ibid., p. 258

9. Cité par P. Santarcangeli, le Livre des labyrinthes: histoire d'un mythe et d'un symbole, Paris, Gallimard, 1974.

10. Ch. Baudoin, op. cit., p. 225. Cf. les remarques de Ph. Borgeaud, à qui j'emprunte l'expression, au demeurant très jungienne, figurant au titre de mon article: "The passage of the labyrinth is a regressive progression... One could speak of a "régression en avant" to the degree that memory (the thread of Ariadne) proclaims a future. This memory is eschatological, a memory-hope. " History of religions, vol. 14, $n^{\circ} 1, p .24$.

11. Montréal, HMH, 1970 , o. 66.

12. L'auteur reprendra cette image: "Mais l'orage mûrissait sous mes aisselles" (p. 93).

13. Cf. P. 93 :

14. P. Santarcangeli, op. cit., p. 219.

15. Cité par Ch. Baudoin, op. cit., p. 219.

16. Cf. MP, P. 104: "s'éveillèrent les dieux amers qu'on traînait avec soi, cavalcade souterraine, sabots de justice, envoútement . On sait que les grands chevaux noirs ( $p$. 87) apparaissent souvent dans les rêves d'angoisse.

17. Voir "le Stade de miroir comme formateur de la fonction de Je" dans les Ecrits de J. Lacan, Paris, Seuil.

18. Dialogue sur la traduction, p. 80.

19. Cf. dans MP: «Que le vent soit pressenti tel un prophète véhément, que l'on respire au centre du cœur...", p. 85.

20. Parlant d'homéopathie, je me permets de signaler à l'attention du lecteur friand de détails curieux ces coutumes que je trouve dans le Larousse du $X X^{\mathrm{e}}$ siecle (vol. 4, 1928, p. 932): "Jadis on employait en pharmacopée la poudre provenant des momies embaumées d'Egypte et à laquelle on attribuait des propriétés merveilleuses contre les blessures et contusions... Les fauconniers administraient en pilules aux oiseaux qui avaient reçu un coup ou attrapé un effort, une "momie" fabriquée avec un vautour dépouillé, séché dans du sable et conservé pendant un an au moins! "...

21. Le mot revient dans le recueil suivant en un contexte éloquent: "Laisse le don alerté mûrir son étrange alchimie en des équipages fougueux " (p. 82).

22. MAS, p. 566 . 